\title{
Recent Progress in Psychosocial and Psychopharmacologic Treatments for ADHD
}

Jeffrey $H$ Newcorn, $M D^{1, *}$

Iliyan Ivanov, $M D^{1}$ Anil Chacko, $P h D^{1,2}$

\author{
Address \\ *,1 Department of Psychiatry, Icahn School of Medicine at Mount Sinai, One \\ Gustave L. Levy PlaceBox 1230, New York, NY 10029, USA \\ Email: jeffrey.newcorn@mssm.edu \\ Emial: iliyan.ivanov@mssm.edu \\ ${ }^{2}$ Steinhardt School of Culture, Education, and Human Development; Department \\ of Applied Psychology, New York University, 246 Greene Street, New York, NY \\ 10003, USA \\ Email: anil.chacko@nyu.edu
}

Published online: 25 February 2015

(C) Springer International Publishing AG 2015

This article is part of the Topical Collection on Child and Adolescent Psychiatry

Keywords ADHD - Psychosocial treatment - Psychopharmacologic treatment

\section{Opinion statement}

In this article, we review several new psychosocial and psychopharmacologic treatment approaches for attention deficit hyperactivity disorder (ADHD). Several of the treatments reviewed are promising and offer opportunities for augmenting current evidence-based approaches. However, others are not yet ready for prime time. Consequently, existing algorithms for ADHD treatment should still be followed. Current guidelines include those from the American Academy of Pediatrics (Wolraich et al. Pediatrics. 128 5:1007-22 2011), the American Academy of Child and Adolescent Psychiatry (AACAP) (Pliszka S and AACAP Work Group on Quality Issues. J Am Acad Child Adolesc Psychiatry. 46 7:894-921 2007), and the Texas Department of Mental Health and Mental Retardation (Pliszka et al. J Am Acad Child Adolesc Psychiatry. 45 6:642-57 2006). These treatment recommendations and algorithms are based on results from both controlled clinical trials and expert opinion. For core symptoms, medication is considered superior to psychosocial treatment. However, the full range of ADHD-associated impairments is best addressed by combined treatment, with an emphasis on behavioral interventions as first-line approaches when treating preschool children with ADHD. The availability of an evidencebased organizational skills training program is a welcome addition for targeting problems 
with organization and attention-related impairments in children. Among available medication treatments, stimulants are considered to have the most robust effects on core symptoms; however several non-stimulant medications are available and others are being investigated for possible utility. Combined pharmacotherapy with stimulants and a2 agonists is now an FDA-approved treatment and can be considered in youth who are not fully responsive to stimulants or who have other problems which may represent indications for $\alpha 2$ agonist treatment. Combined treatment with stimulants plus risperidone has merit in youth with severe aggressive behavior but remains off-label and should generally be considered when other options fail. The potential risk and benefits of stimulant treatment for subsequent substance abuse remains an important topic. In the main, stimulant treatment is considered safe and may even provide protection against development of substance abuse. However, the possibility that stimulant exposure could be problematic in youth with elevated risk for substance abuse disorders should be considered.

\section{Introduction}

Attention deficit hyperactivity disorder (ADHD) is a highly prevalent and heterogeneous condition, and numerous evidence-based psychopharmacologic and psychosocial treatments are available. In this article, we consider several issues related to existing and emerging treatments in ADHD, and highlight findings from selected recent clinical trials and treatment approaches of interest, focusing on both psychosocial and pharmacologic approaches. We examine the following topics: (1) development of an evidence-based organizational training program for older children and adolescents; (2) recent studies examining the impact of "brain training" on ADHD symptoms; (3) rationale for non-stimulant pharmacotherapy and considerations regarding possible effects of stimulants and non-stimulants on substance abuse risk; (4) recent findings and reviews of $\alpha 2$ agonists administered as monotherapy or adjunctive to stimulants; and (5) combined treatment in youth with ADHD and severe aggression.

\section{Psychosocial treatment}

Evans and colleagues (2014) categorize psychosocial treatments as either behavioral management interventions [ $4 \bullet$ ] (which aim to teach individuals other than the patient skills required to modify the patient's behavior in context) or training interventions (which focus directly on the patient to teach skills that improve behavior and adaptive function). Behavior management interventions, including behavioral parent training, behavioral classroom management, and behavioral peer interventions-all of which are primarily used with preschool through adolescents with ADHD-have the most robust empirical support among the various psychosocial treatments for ADHD [4•] (see Evans, Owens, \& Bunford, 2014 for a recent review). However, there are notable limitations to these treatments, particularly the lack of normalization, generalization to nontreatment settings, and sustained effects over time. These shortcomings have led to investigation of alternative psychosocial treatments, with a particular focus on training interventions. Although there are numerous training interventions (e.g., traditional social skills interventions), we 
focus our attention on three of the most theoretically promising, which include organizational skills training, neurocognitive treatments, and neurofeedback.

Organizational skills training interventions have been developed for school-age and middle-school age youth, for use in clinic [5•] and school [6] settings. These interventions focus on teaching children new tools and routines to record assignments, organize school materials, more effectively monitor the amount of time involved in completing assignments, and break larger tasks into smaller more manageable ones. Often, parents and teachers are taught to reinforce children for making an effort to use the organizational approaches. Development of organizational training programs for youth is theoretically important for older school-age youth and adolescents with ADHD, as organizational capacity is often required for developmentally relevant tasks in these age groups, and older children with ADHD have considerable difficulty in this area relative to non-ADHD peers. Heretofore, such skill development and training programs have only been available for adults with ADHD.

Results of recent randomized clinical trials demonstrate that youth receiving organizational skills training have significantly improved academic functioning and homework completion, less family conflict, and better ratings of organization as reported by parents [5•, 2012; 6]. Teachers also report significant improvements in organization [5•], although this has been less consistently observed [6]. To maximize the effects of organizational skills training, parent and teachers must collaborate and support youth to utilize the skills they have learned. While the results of these two recent studies are encouraging, replication is needed in more diverse samples and settings.

Since the seminal work conducted by Klingberg and colleagues (2005) [7] suggested that neurocognitive training (i.e., Cogmed Working Memory Training; CWMT) could improve both the targeted neurocognitive deficit (i.e., working memory) associated with ADHD as well as ADHD outcomes (e.g., ADHD symptoms), there has been a substantial interest in the evaluation of neurocognitive interventions. Since 2005, there have been over 25 published studies in this area. While a review of each of these studies is beyond the scope of this paper, we focus on CWMT as an exemplar, given that the majority of neurocognitive treatment studies of ADHD in youth as well as many of the limitations and issues regarding neurocognitive training have focused on CWMT.

Initially, multiple RCTs [8, 2010;9] demonstrated some benefit ofCWMT, primarily on parent-rated ADHD symptoms and working memory tasks. However, reviews of the CWMT literature [10] have suggested limitations 
regarding the external and internal validity of previous studies. In the largest study of CWMT in youth with ADHD, Chacko and colleagues (2014) addressed many of the limitations in previous studies [11•]. Findings indicate benefits of CWMT on some aspects of working memory function, but not on parent/teacher-reports of ADHD symptoms, objective measures of attention, impulsivity and activity, or academic achievement outcomes. The lack of significant effects of CWMT on ADHD symptoms is in keeping with findings from other studies of neurocognitive interventions for youth (school-age through adolescence)

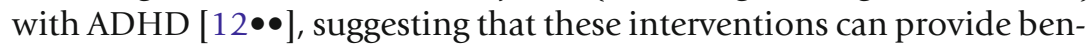
efit for targeted aspects of executive functioning (e.g., short term memory) but not on ADHD outcomes. Collectively, although the results of recent studies and meta-analyses are sobering, the data suggest that neurocognitive interventions can improve targeted functions. As such, there is promise that novel interventions could potentially be developed to alter specific neurocognitive functions which constitute manifestations of the underlying pathophysiology of ADHD, resulting in downstream effects on broader outcomes (e.g., ADHD symptoms; academic functioning), and perhaps, more enduring effects of treatment. This remains a high priority area of investigation; however; existing data suggest that current neurocognitive interventions should not be considered as viable treatments for ADHD in youth.

Neurofeedback training has undergone increasingly rigorous evaluation over the past several years. Early studies of neurofeeedback suggested moderate to large effects on ADHD symptoms; however, these studies were often criticized for using less rigorous methods (e.g., nonrandomization to treatment; unreliable or subjective outcome measures) and poor diagnostic procedures for study inclusion. Recent, more rigorous studies have been conducted, albeit with mixed results. Beneficial effects on ADHD symptoms have been reported in studies using reporters who were likely not blind to treatment condition; however, findings in studies with raters more likely blind to treatment condition less consistently demonstrate benefit [13-18]. The lack of consistent effects on blinded outcomes is particularly problematic for interventions such as neurofeedback, which aim to produce effects that generalize across settings, and which should be stable over time (see Chacko et al. 2013, for further discussion of this point) [10]. Interestingly, data suggest some stability of effects following acute neurofeedback treatment $[17,19]$. Findings also suggest that neurofeedback is as effective as stimulant medication on ADHD symptoms [19, 20], although this is not the case in all studies [21]. Importantly, for studies suggesting equivalence between neurofeedback and medication, medication was administered as a fixed dose, standardized based on body weight, rather than titrated to optimal dose. As this approach likely results in under-dosing for at least some subjects, these studies do not compare neurofeedback to optimally prescribed medication. 


\section{Pharmacotherapy}

To summarize, neurofeedback has garnered some promising support as an intervention for the treatment of ADHD. However, further, well-controlled studies in comparison to alternative, well-implemented first-line pharmacological (i.e., stimulant medication) and nonpharmacological (i.e., behavioral treatment) interventions are clearly necessary before neurofeedback can be established as an evidence-based treatment option for ADHD.

Rationale for non-stimulant medication

An ever-growing basic science and translational research database has evaluated the scientific basis of response to psychostimulants, and an extensive clinical research literature has established these medications as first-line treatments for ADHD in both children and adults. In addition, recent research has indicated the functional benefits of stimulant treatment [22]. Yet, despite the very high response rates, up to $30 \%$ of patients will not respond optimally, and an additional $4-5 \%$ may experience disabling side effects leading to treatment discontinuation. Furthermore, stimulants are class B controlled substances, and although the majority of research indicates that, on average, stimulants do not increase risk for development of substance use disorders (SUD), results of other studies still raise questions regarding the possibility of increased risk. In addition, there are more specific concerns about the effects of stimulants in high-risk individuals, or those with a positive family history of substance use disorders. Finally, stimulants are inherently short-acting medications, and even the new longacting medications which dominate the treatment landscape rarely produce consistent effects throughout the entire day. Consequently, there has been increased interest in developing non-stimulant agents that may offer symptomatic and functional benefits without the complicating issues regarding substance use risk, and ideally with a better time-action profile than the stimulants. Atomoxetine (ATX) has been available in the US since 2002 and, similar to stimulants, has been extensively studied. Although the overall effects are not as robust as they are for stimulants, some individuals respond to or tolerate this medication better than stimulants [23, 24]. Use of atomoxetine in stimulant non-responders is at least theoretically supported by research indicating both shared and distinct mechanisms of action of ATX and stimulants [25, 26•]. Moreover, many (but not all) individuals who respond to stimulants also respond to ATX, suggesting that either treatment could be used.

The relationship of ADHD treatment and comorbid SUD has been examined in several important recent studies. As stimulants strongly enhance the release of dopamine in brain regions associated with reward processing and motivation, the use of stimulants in patients with past substance use has 
raised continuous clinical concerns, both in terms of possible misuse of stimulants and because of possible "sensitization" effects of stimulant exposure and consequent increased risk for relapse of the primary substance use disorder. One meta- analysis, including data from 15 studies $(n=2,565)$, found neither increased risk nor protective effects of stimulant treatment in

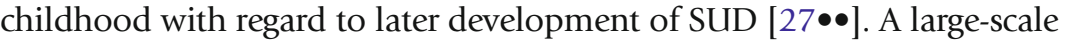
retrospective study using the Swedish national registers examined individuals born between 1960 and 1998 and diagnosed with ADHD (male $n=26,249$; female $n=12,504$ ) and found that there may be protective effects of stimulants with respect to later SUD [28]. Furthermore, one recent study reported no onset of new SUD for a group of ADHD patients without comorbid SUD at baseline who were treated with stimulants for 41.1 months, while $58.5 \%$ of patients with ADHD and comorbid SUD at baseline showed one or more relapses of SUD during treatment with stimulants (mean length, 27.9 months) [29•, 30]. The latter findings raise the question of whether stimulant treatment could be a possible mediator of SUD relapse in individuals with ADHD and comorbid SUD, and if so, what the neurobiological basis for this might be. Finally, one clinical report by Wilens et al. (2011) showed that atomoxetine treatment was associated with clinical improvement in ADHD symptoms, which correlated significantly with a decrease in alcohol craving. However, in the placebo-treated group, there was greater relapse to alcohol abuse, which significantly correlated with worsening of ADHD symptoms. It is worth mentioning that, although atomoxetine does have direct effects on dopamine in PFC via action of norepinephrine transporters [25], its influence on dopaminergic activation is predominantly seen in cortical brain regions, as opposed to the dopamine-enhancing effects of stimulants in basal ganglia.

To summarize, we continue to see conflicting findings from large-scale meta-analyses and registry studies that are challenging to integrate into a single coherent message regarding stimulant treatment of ADHD and substance abuse. Although we tend to think this means we don't fully understand the relationship, if there is one, maybe the conflicting findings arise because there isn't a single answer to this question. We therefore pose the following unanswered question as a topic for future research: Might selected subgroups of individuals with ADHD and high risk for substance abuse or prior SUD be at heightened risk from stimulant treatment, and if so, can these patients be treated successfully with non-stimulant agents with a lower risk for substance use development or relapse?

Interest in the $\alpha 2$-adrenoceptor agonists, clonidine, and guanfacine as treatments for ADHD has resulted in a rapid expansion of randomized clinical trials over the past several years, primarily linked to the development of new long-duration formulations of these agents. The alpha- 2 agonists have a relatively long half-life, but the duration of behavioral activity of the immediate release formulations is shorter. To address this limitation, the new long-acting formulations increase the time to maximal concentration (Tmax), with peak activity reached at 5-6 h, which accounts for their more sustained clinical effects. 
Several recent publications have examined data from randomized clinical trials for monotherapy and adjunctive therapy with $\alpha 2$ agonists. Sallee et al. (2013) reviewed studies for both clonidine and guanfacine conducted up until October 2012, finding that all available immediate-release and longacting formulations of clonidine and guanfacine are effective for ADHD symptoms when administered either as monotherapy, or adjunctive therapy with psychostimulants [31•]. It is noted that, although adjunctive therapy with psychostimulants may provide additional clinical benefit compared with psychostimulants alone, the magnitude of this benefit is understandably smaller than with monotherapy. Also to be noted is that the majority of studies examining adjunctive treatment do not require subjects to have optimal stimulant dosing, which might minimize or even eliminate the need for adjunctive treatment. On the other hand, the potential value of $\alpha 2$ agonist augmentation on stimulant dose is illustrated by findings from one study [32], in which $15 \%$ of participants treated with clonidine extended-release plus stimulant were able to reduce their psychostimulant dose. The effect size favoring adjunctive therapy with GXR over monotherapy was recently estimated to be approximately 0.4 [33]. Additional clinical wisdom regarding the use of guanfacine extended release comes from post hoc findings suggesting a relationship between weight and optimal dose (approximately $0.08 \mathrm{mg} / \mathrm{kg}$ ), with better established efficacy shown in children than adolescents (who may need higher weight-adjusted doses) [34, 35]. Also, recent evidence suggests that the medication can be given either in the morning or evening, with comparable clinical effects [24] and benefit which is more or less consistently maintained throughout the day [36].

Two large-scale meta-analyses of $\alpha 2$ agonists for the treatment of ADHD have recently been published. One European publication examined the safety and efficacy of guanfacine (but not clonidine), based on data from seven randomized controlled trials (including US studies) with a total 1,752 participants, including data from papers published up to May, 2014 [37]. A score of $\leq 2$ (i.e., much or very much improved) on the Clinical Global Impressions Improvement scale (i.e., CGI-I) served as the primary definition of response for the meta-analysis, to examine response based on a categorical measure of improvement rather than on symptom reduction. The $59.0 \%$ of the participants in the guanfacine group $(n=1,177)$ benefited from the treatment compared with $33.3 \%$ in the placebo group $(n=575)$. The number of participants with at least one adverse event were 948 $(82.4 \%)$ in the guanfacine group and $376(67.9 \%)$ in the placebo group (OR 2.6; 95\%CI 1.6-4.4), with somnolence, sedation, and fatigue being most frequent. The authors conclude that guanfacine can be considered safe and effective for children and adolescents with ADHD. However, they also noted the relative absence of comparator data, making it impossible to assess comparative efficacy and render clinical decisions about how and when to preferentially use this medication in clinical practice.

Another meta-analysis examined the utility of both clonidine and guanfacine in randomized trials published until May 2013 [38] and included a total of 12 studies $(N=2,276)$, nine for monotherapy $(n=1,550)$ and three for adjunctive therapy $(n=726)$. Reduction of overall ADHD symptoms was the primary outcome, with reduction in hyperactivity/ 
impulsivity, inattentiveness, oppositional defiant disorder symptoms, allcause and specific-cause discontinuation, and adverse effects as secondary outcomes. Monotherapy significantly reduced overall ADHD symptoms (SMD $=-0.59, p<0.00001)$ as well as all secondary outcome measures. These effects were similar, although less robust ( $\mathrm{SMD}=-0.36, p<0.0001)$ in adjunctive trials. Monotherapy was associated with lower all-cause $(\mathrm{RR}=0.70, p=0.01$, number-needed-to-treat $(\mathrm{NNT})=10)$ and inefficacyrelated $(\mathrm{RR}=0.39, p<0.0001)$ discontinuations versus placebo but also significantly more fatigue (number-needed-to-harm $(\mathrm{NNH})=10)$, sedation $(\mathrm{NNH}=17)$, and somnolence $(\mathrm{NNH}=4)$ and significantly greater hypotensive (clonidine-IR), bradycardic (clonidine-IR), and QTc prolonging (guanfacine-XR) cardiac effects. However, discontinuation rates due to side effects were similar for the active drug groups and the placebo-treated participants. In adjunctive trials, the all-cause and specific-cause discontinuations with $\alpha 2$ agonists were comparable to those of placebo.

Non-stimulant augmentation of stimulant treatment in youth with aggression

Several studies have examined the potential role of medications used to treat mood or psychotic disorders to augment psychostimulant response in youth with ADHD+ODD/CD or aggression. Blader et al. (2009) evaluated the efficacy of divalproex for reducing aggressive behavior among children 6 to 13 years of age with ADHD and a disruptive disorder whose chronic aggression was under-responsive to psychostimulant treatment alone [39]. A higher percent of children ( $57 \%$ ) who were randomly assigned to augmentation with divalproex showed improved aggression compared with children assigned to placebo (15\%). However, subsequent reports from the same group also suggest that optimization of stimulant doses (i.e., OROS$\mathrm{MPH}$ in doses up to $90 \mathrm{mg}$ daily) may adequately address overt aggression in children with ADHD and comorbid ODD or CD without addition of a second medication [40 $\bullet$. Moreover, new findings indicate that the presence of proactive aggression and callous-unemotional traits are not necessarily associated with limited response to optimized stimulant regimen [41], as has previously been thought to be the case. In this study, treatment produced improvement in both callous-unemotional traits and proactive aggression, similar to what was observed for other behavioral indicators. Additional agents that have been used clinically to address aggressive behaviors in youth with ADHD include second-generation antipsychotics. However, there has been relatively limited evidence supporting efficacy for this indication relative to their fairly extensive clinical use. One recent study examined the effects of risperidone adjunctive to stimulants in 168 children 6 to 12 years of age with ADHD and severe physical aggression who were treated with stimulants and were provided behavioral parent training therapy. Half of the participants were randomized to a 9-week trial of parent training, stimulant, and placebo (basic treatment; $n=84$ ) or parent training, stimulant, and risperidone (Augmented treatment; $n=84$ ) [42]. Findings indicate that risperdal augmentation provided moderate but variable improvement in reducing aggressive and other seriously disruptive behaviors. Another recent publication conducted a retrospective review of 
44 cases treated with $\mathrm{MPH}+$ risperidone [43], concluding that this combined treatment, despite tolerability concerns (weight gain $n=31$, tachycardia $n=3$, dyskinesia $n=1$ ), may be particularly efficacious for children with $\mathrm{ADHD}$ and comorbid CD [34]. Another recent review examined the potential utility of aripiprazole adjunctive to stimulants in ADHD+aggression from a total of 34 publications (although only two were randomized trials) and reported mostly negative findings [44]. Together, these new publications indicate the potential importance of adjunctive treatments with mood-stabilizing or antipsychotic agents in the management of ADHD+aggression, with the data thus far favoring divalproex and risperidol, although such an approach should be balanced against the potential for relatively serious side effects (e.g., weight gain, sedation, other), the recognition that well-titrated monotherapy may obviate the need for combined treatment, and the potential availability of other agents (e.g., $\alpha 2$ agonists) to augment response.

Several recently published studies and reviews highlight the potential role of dietary management or nutritional supplementation in the management of ADHD. A large number of dietary supplements have been studied, including minerals (e.g., zinc and iron), vitamins, caffeine, St John's Wort, bark extracts, amino acids (e.g., tyrosine, tryptophan), carnitine, and aspartame. A recent review and meta-analysis of randomized clinical trials (RCT) [45•] reported that, although some supplements such as zinc, carnitine, caffeine, tryptophan, and St. John's Wort have shown significant improvement on some outcome measures (e.g., parent or teacher ratings) in some clinical trials, there has been a lack of consistent findings for any of the supplements showing robust and significant reduction of ADHD symptoms. For other supplements such as iron, tyrosine, aspartate, and vitamins, findings have largely been negative. Relatively more consistent results were noted for three dietary treatments, all of which have been tested in at least one RCT: (1) restricted elimination diets (RED), which entails the removal of foods to which the child may show either allergic (i.e., IgE-mediated) or nonallergic hypersensitivity; (2) artificial food colors exclusion (AFCE); and (3) supplementation with free fatty acids (SFFA), based on reports that children with ADHD show deficiencies in free fatty acids. This comprehensive review concluded that well-conducted studies provided most consistent evidence for a small effect of SFFA. While RED and AFCE may be beneficial and potentially valuable treatments, large-scale studies are needed to determine their effect size and identify types of children who may benefit most. The omega-3 fatty acids docosahexaenoic acid (DHA) and eicosapentaenoic acid (EPA) are orthomolecular, conditionally essential nutrients that function exclusively at the level of the cell membranes [46]. DHA is essential to pre- and postnatal brain development, whereas EPA seems to influence mood and behavior. Both substances also generate neuroprotective metabolites. Combinations of DHA and EPA have shown benefits in controlling cognitive deficits, mood liability, and aggression in several psychiatric conditions. Manor et al. [47] evaluated the safety of phosphatidylserine attached to a combination of DHA/EPA (omega-3 PS) in 200 children with 
ADHD, who were randomized to receive either 300 mg PS-omega 3 or placebo for 15 weeks. There were no significant differences between the two active treatment and placebo groups in any of the safety parameters evaluated. The authors reported significant reduction in the Global-Restless/ impulsive subscale of Conners' Rating Scale, Parent Version (CRS-P) and the significant improvement in Parent impact-emotional (PE) subscale of the Child Health Questionnaire (CHQ) [48]. An exploratory analysis conducted in a subgroup of children with more pronounced hyperactive/ impulsive behavior, as well as mood and behavioral dysregulation, revealed a significant reduction in the ADHD-Index and subscales measuring hyperactivity. Efficacy was maintained in an open-label extension study, and children who switched to PS-omega3 treatment from placebo also showed a significant reduction in subscales scores on both the CRS-P and Conners' Teacher Rating Scale (CRS-T). However, results of another recent RCT on the effects of EPA/DHA supplementation in 95 children 6-12 years of age diagnosed with ADHD [49] — using a different formulation of EPA/ DHA-failed to demonstrate significant improvements in ADHD symptoms. Another study examined comparative efficacy and tolerability of omega 3/6 fatty acids and MPH administered individually and in combination in 90 children with ADHD for 12 months [50]. Findings indicate that the combination treatment was associated with significantly better outcomes on the ADHD Total and Hyperactivity-Impulsivity subscales compared with omega-3/6 fatty acids alone, although results on the Inattention subscale were similar. Furthermore, the combination of omega- $3 / 6$ fatty acids and MPH had similar effects to MPH alone. However, there were some tolerability benefits for the combination treatment.

To summarize, a number of studies have examined the potential role of dietary management and nutritional supplementation in ADHD treatment. Many of these investigations yielded negative or inconclusive findings. However, there are positive findings in some studies (most notably with certain dietary restrictions, and supplementation with omega $3 / 6$ fatty acid formulations); hence, more extensive study is underway. It is not yet possible to determine how and when these interventions should be used, and which patients might be most likely to respond. Hence, no changes to existing treatment algorithms can be recommended at present.

\section{Summary and discussion}

This article has reviewed recent selected clinical trials, reviews, and metaanalyses of psychosocial, pharmacological, and nutraceutical treatments for ADHD, evaluating the strength of evidence for or against a variety of novel approaches. In each case, there appears to be opportunity, but, at the same time, all of the new and/or investigational interventions have limitations. In particular, we highlight the beneficial effects of several psychosocial treatments on targeted rather than global outcomes and the need for more rigorous methodology in future trials. Importantly, several of the psychosocial interventions discussed target etiological or key compensatory mechanisms linked to the 
development and/or persistence of ADHD, thereby offering the promise of generalized and sustained effects not necessarily associated with other interventions. Therefore, despite the modest findings to date, this line of research remains very important. The overall conclusions regarding non-stimulant medications and nutraceutical agents are fairly similar, with the greatest benefits seen for selected outcomes or in specific subgroups of patients. With regard to medications, non-stimulants are effective when used in monotherapy, though they have lower overall efficacy than stimulants. Nevertheless, possible decreased risk for substance abuse development in high-risk patients or relapse in patients with prior SUD (atomoxetine), augmentation of stimulant effects with the possibility of lower stimulant dose ( $\alpha 2$ agonists), and treatment of severe aggression in context of ADHD (divalproex, risperidone) all represent potentially important targeted uses. Some nutritional supplements (e.g., omega $3 / 6$ fatty acid formulations) have shown benefit in some studies, most evident in selected subgroups of patients, and with a favorable tolerability profile. However, there is need for larger-scale studies to estimate their efficacy in controlling ADHD symptoms.

In conclusion, results of recent studies examining new psychosocial, pharmacological, and nutraceutical treatment approaches show potential to enhance the therapeutic armamentarium for ADHD and related symptom domains, with particular relevance for the treatment of selected subgroups. The development of new and better targeted psychosocial and pharmacological approaches remains a high priority for the field.

\section{Compliance with Ethics Guidelines}

\section{Conflict of Interest}

Jeffrey Newcorn has received research support from Shire Pharmaceuticals, serves on a DSMB for Sunovion, and is an advisor and/or consultant for Alcobra, Biobehavioral Diagnostics, GencoSciences, Ironshore, Lupin, Neurovance, and Shire.

Iliyan Ivanov is a member of the Data Safety Monitoring Board for Lundbeck.

Anil Chacko has no conflicts of interest to report.

Human and Animal Rights and Informed Consent

This article does not contain any studies with human or animal subjects performed by any of the authors.

\section{References and Recommended Reading}

Papers of particular interest, published recently, have been highlighted as:

- Of importance

$\bullet \quad$ Of major importance

1. Wolraich M, Brown L, Brown RT, DuPaul G, Earls M, Feldman HM, et al. Subcommittee on Attention-Deficit/Hyperactivity Disorder; Steering Committee on Quality Improvement and Management. ADHD: clinical practice guideline for the diagnosis, evaluation, and treatment of attention-deficit/hyperactivity disorder in children and adolescents. Pediatrics. 2011;128(5):1007-22. 
2. Pliszka S, AACAP Work Group on Quality Issues. Practice parameter for the assessment and treatment of children and adolescents with attention-deficit/hyperactivity disorder. J Am Acad Child Adolesc Psychiatry. 2007;46(7):894-921.

3. Pliszka SR, Crismon ML, Hughes CW, Corners CK, Emslie GJ, Jensen PS, et al. Texas consensus conference panel on pharmacotherapy of childhood attention deficit hyperactivity disorder. The Texas Children's Medication Algorithm project: revision of the algorithm for pharmacotherapy of attention-deficit/hyperactivity disorder. J Am Acad Child Adolesc Psychiatry. 2006;45(6):642-57.

4. $\quad$ Evans SW, Owens JS, Bunford N. Evidence-based psychosocial treatments for children and adolescents with attention-deficit/hyperactivity disorder. J Clin Child Adolesc Psychol. 2014;43:527-51.

Most recent review of psychosocial interventions for the treatment of ADHD per Society of Clinical Child and Adolescent Psychology's evidence-based criteria.

5.• Abikoff H, Gallagher R, Wells KC, et al. Remediating organizational functioning in children with ADHD: immediate and long-term effects from a randomized controlled trial. J Consult Clin Psychol. 2012;81:11328.

Randomized controlled trial investigating contingency-based versus skill-based psychosocial treatment to address organization, time management, and planning behavior in youth with ADHD. This study is likely the most rigorous evaluation of organizational skills treatment and suggest that this intervention can improve outcomes with maintenance of gains over time.

6. Langberg JM, Epstein JN, Becker SP, et al. Evaluation of the homework, organization, and planning skills (HOPS) intervention for middle school students with attention deficits hyperactivity disorder as implemented by school mental health providers. School Psychol Rev. 2012;41:342-64.

7. Klingberg T, Fernell E, Olesen PJ, et al. Computerized training of working memory in children with ADHD - a randomized, controlled trial. J Am Acad Child Adolesc Psychiatry. 2005;44(2):177-86.

8. Beck SJ, Hanson CA, Puffenberger SS, et al. A controlled trial of working memory training for children and adolescents with ADHD. J Clin Child Adolesc Psychol. 2010;39:825-36. doi:10.1080/15374416.2010. 517162.

9. Green CT, Long DL, Green D, Iosif AM, Dixon JF, Miller $\mathrm{MR}$, et al. Will working memory training generalize to improve off-task behavior in children with attentiondeficit/hyperactivity disorder? Neurotherapeutics. 2012;9(3):639-48.

10. Chacko A, Feirsen N, Marks D, et al. Cogmed working memory training for youth with ADHD: a closer examination of efficacy utilizing evidence-based criteria. J Clin Child Adolesc Psychol. 2013;42:769-83.

11. Chacko A, Bedard A, Marks D, et al. A randomized clinical trial of Cogmed working memory training in school-age children with ADHD: a replication in a diverse sample using a control condition. J Child Psychol Psychiatry. 2014;55:247-55.

The most rigorous randomized controlled trial of Cogmed Working Memory Training in youth with ADHD. Findings highlight the importance of various non-specific factors in explaining previously documented effects of Cogmed Working Memory Training in youth with ADHD and suggested limited utility of this intervention for youth with ADHD.

12.• Rapport MD, Orban SA, Kofler MJ, et al. Do programs designed to train working memory, other executive functions, and attention benefit children with ADHD: a meta-analytic review of cognitive, academic, and behavioral outcomes. Clin Psychol Rev. 2013;33:123752.

Recent meta-analysis of various brain training interventions for ADHD. Findings suggest that these intervention improve some aspects of executive functioning (e.g., short-term memory), there is little support that the interventions analyzed have substantial effects on key executive functions associated with ADHD (e.g., working memory; sustained attention).

13. Lansbergen MM, van Dongen-Boomsma M, Buitelaar JK, et al. ADHD and EEG-neurofeedback: a doubleblind randomized placebo-controlled feasibility study. J Neural Transm. 2011;118(2):275-84.

14. Maurizio S, Liechti $\mathrm{M}$, Heinrich $\mathrm{H}$, et al. Comparing tomographic EEG neuro-feedback and EMG biofeedback in children with attention-deficit/hyperactivity disorder. Biol Psychol. 2014;95:31-44.

15. Steiner NJ, Sheldrick RC, Gotthelf D, Perrin E. Computer-based attention training in the schools for children with attention deficit/hyperactivity disorder: a preliminary trial. Clin Pediatr. 2011;50:615-22.

16. Steiner NJ, Frenette EC, Rene KM, et al. Neurofeedback and cognitive attention training for children with attention-deficit hyperactivity disorder in schools. J Dev Behav Pediatr. 2014;35(1):18-27.

17. Steiner NJ, Frenette EC, Rene KM, et al. In-school neurofeedback training for ADHD: sustained improvements from a randomized control trial. Pediatrics. 2014;133(3):483-92.

18. Van Dongen-Boomsma M, Vollebregts MA, SlaatsWillemse D, et al. A randomized placebo-controlled trial of electroencephalographic (EEG) neurofeedback in children with attention-deficit/hyperactivity disorder. J Clin Psychiatry. 2013;74(8):821-7.

19. Meisel V, Servera M, Garcia-Banda G, et al. Neurofeedback and standard pharmacological intervention in ADHD: a randomized controlled trial with six-month follow-up. Biol Psychol. 2013;94(1):1221.

20. Duric N, Assmus J, Gundersen D, et al. Neurofeedback for the treatment of children and adolescents with ADHD: a randomized and controlled clinical trial using parental reports. BMC Psychiatry. 2012;12(1):107.

21. Ogrim G, Hestad KA. Effects of neurofeedback versus stimulant medication in attention-deficit/hyperactivity disorder: a randomized pilot study. J Child Adolesc Psychopharmacol. 2013;23(7):448-57. 
22. Banaschewski T, Soutullo C, Lecendreux M, Johnson M, Zuddas A, Hodgkins P, et al. Health-related quality of life and functional outcomes from a randomized, controlled study of lisdexamfetamine dimesylate in children and adolescents with attention deficit hyperactivity disorder. CNS Drugs. 2013;27(10):829-40.

23. Newcorn J, Kratochvil C, Allen AJ, Casat C, Ruff D, Moore $\mathrm{R}$, et al. Atomoxetine and osmotically released methylphenidate for the treatment of attention deficit hyperactivity disorder: acute comparison and differential response. Am J Psychiatry. 2008;165(6):721-30.

24. Newcorn JH, Stein MA, Childress AC, Youcha S, White C, Enright G, et al. Randomized, double-blind trial of guanfacine extended release in children with attentiondeficit/hyperactivity disorder: morning or evening administration. J Am Acad Child Adolesc Psychiatry. 2013;52(9):921-30.

25. Bymaster FP, Katner JS, Nelson DL, Hemrick-Luecke SK, Threlkeld PG, Heiligenstein JH, et al. Atomoxetine increases extracellular levels of norepinephrine and dopamine in prefrontal cortex of rat: a potential mechanism for efficacy in attention deficit/ hyperactivity disorder. Neuropsychopharmacology. 2002;27(5):699-711.

26. Schulz KP, Fan J, Bédard A-C, Clerkin S, Ivanov I, Tang $\mathrm{C}$, et al. Common and unique therapeutic mechanisms of stimulant and non-stimulant treatments for ADHD. Arch Gen Psychiatry. 2012;69(9):952-61.

The first neuroimaging study to identify different meachnisms of action for stimulant and non-stimulant medications and their relation to clinical improvement of ADHD symptoms.

27.• Humphreys KL, Eng T, Lee SS. Stimulant medication and substance use outcomes: a meta-analysis. JAMA Psychiatry. 2013;70(7):740-9.

Large scale meta-analysis of stimulant use and risk for subsequent substance abuse finds that, overall, there is neither increased nor decreased risk.

28. Chang Z, Lichtenstein P, Halldner L, D'Onofrio B, Serlachius E, Fazel S, et al. Stimulant ADHD medication and risk for substance abuse. J Child Psychol Psychiatry. 2014;55(8):878-85.

29. Torgersen T, Gjervan B, Rasmussen K, Vaaler A, Nordahl HM. Prevalence of comorbid substance use disorder during long-term central stimulant treatment in adult ADHD. Atten Defic Hyperact Disord. 2013;5(1):59-67.

The first reports to demonstrate that adult individuals with $\mathrm{ADHD}$ and comorbid substance use might be at elevated risk for substance use relapse while receving stimulant tretment for ADHD.

30. Wilens TE, Adler LA, Tanaka Y, Xiao F, D'Souza DN, Gutkin SW, et al. Correlates of alcohol use in adults with ADHD and comorbid alcohol use disorders: exploratory analysis of a placebo-controlled trial of atomoxetine. Curr Med Res Opin. 2011;27(12):230920.

31. Sallee F, Connor DF, Newcorn JH. A review of the rationale and clinical utilization of $\alpha 2$-adrenoceptor agonists for the treatment of attention-deficit/ hyperactivity and related disorders. J Child Adolesc Psychopharmacol. 2013;23(5):308-19.

In depth review of clinical studies of immediate- and extendedrelease $\alpha 2$-adrenoceptor agonists (e.g. clonidine and guanfacine) showing clinical efficacy and safety of these agents as monotherapy and adjunctive therapy with

psychostimulants for the symptomatic treatment of ADHD.

32. Kollins SH, Jain R, Brams M, Segal S, Findling RL,

Wigal SB, et al. Clonidine extended-release tablets as add-on therapy to psychostimulants in children and adolescents with ADHD. Pediatrics.

2011;127(6):e1406-13.

33. Wilens TE, Bukstein O, Brams M, Cutler AJ, Childress A, Rugino $\mathrm{T}$, et al. A controlled trial of extended-release guanfacine and psychostimulants for attention-deficit/ hyperactivity disorder. J Am Acad Child Adolesc Psychiatry. 2012;51(1):74-85.

34. Biederman J, Melmed RD, Patel A, McBurnett K, Konow J, Lyne A, et al. A randomized, double-blind, placebo-controlled study of guanfacine extended release in children and adolescents with attention-deficit/ hyperactivity disorder. Pediatrics. 2008;121(1):e7384.

35. Sallee FR, McGough J, Wigal T, Donahue J, Lyne A, Biederman J, et al. Guanfacine extended release in children and adolescents with attention-deficit/hyperactivity disorder: a placebo-controlled trial. J Am Acad Child Adolesc Psychiatry. 2009;48(2):155-65.

36. Young, J, Rugino, T, Dammerman, R, Lyne, A, Newcorn, J. Efficacy of guanfacine extended release assessed during the morning, afternoon, and evening using a modified Conners' Parent Rating Scale-Revised: short form. J Child Adolesc Psychopharmacol. 2014.

37. Ruggiero S, Clavenna A, Reale L, Capuano A, Rossi F, Bonati M. Guanfacine for attention deficit and hyperactivity disorder in pediatrics: a systematic review and meta-analysis. Eur Neuropsychopharmacol. 2014. pii: S0924-977X(14)00228-4.

38. Hirota T, Schwartz S, Correll C. Alpha-2 agonists for attention-deficit/hyperactivity disorder in youth: a systematic review and meta-analysis of monotherapy and add-on trials to stimulant therapy. J Am Acad Child Adolesc Psychiatry. 2014;53(2):153-73.

39. Blader JC, Schooler NR, Jensen PS, Pliszka SR, Kafantaris V. Adjunctive divalproex versus placebo for children with ADHD and aggression refractory to stimulant monotherapy. Am J Psychiatry. 2009;166(12):1392-401.

40. Blader JC, Pliszka SR, Jensen PS, Schooler NR, Kafantaris V. Stimulant-responsive and stimulant-refractory aggressive behavior among children with ADHD. Pediatrics. 2010;126(4):e796-806.

Examines stimulant treatment of ADHD symptoms and aggression in children with ADHD with and without callous and unemotional traits. Contrary to what might have been expected, both groups responded to stimulant treatment, probably because children with callous and unemotional traits also have impulsive aggression. 
41. Blader JC, Pliszka SR, Kafantaris V, Foley CA, Crowell JA, Carlson GA, et al. Callous-unemotional traits, proactive aggression, and treatment outcomes of aggressive children with attention-deficit/hyperactivity disorder. J Am Acad Child Adolesc Psychiatry. 2013;52(12):1281-93.

42. Aman MG, Bukstein OG, Gadow KD, Arnold LE, Molina BS, McNamara NK, et al. What does risperidone add to parent training and stimulant for severe aggression in child attention-deficit/hyperactivity disorder? J Am Acad Child Adolesc Psychiatry. 2014;53(1):47-60.

43. Javelot H, Glay-Ribau C, Ligier F, Weiner L, Didelot N, Messaoudi $\mathrm{M}$, et al. Methylphenidate-risperidone combination in child psychiatry: a retrospective analysis of 44 cases. Ann Pharm Fr. 2014;72(3):164-77.

44. Ghanizadeh A. Systematic review of clinical trials of aripiprazole for treating attention deficit hyperactivity disorder. Neurosciences. 2013;18(4):323-9.

45. Buitelaar J, Cortese S, Ferrin M, Konofal E, Lecendreux M, Simonoff E, et al. Research review: the role of diet in the treatment of attention-deficit/hyperactivity disorder-an appraisal of the evidence on efficacy and recommendations on the design of future studies. J Child Psychol Psychiatry. 2014;55(5):416-27.

Excellent review of dietary management and use of nutritional supplements for ADHD.

46. Kidd PM. Omega-3 DHA and EPA for cognition, behavior, and mood: clinical findings and structural- functional synergies with cell membrane phospholipids. Altern Med Rev. 2007;12(3):207-27.

47. Manor I, Magen A, Keidar D, Rosen S, Tasker H, Cohen $\mathrm{T}$, et al. The effect of phosphatidylserine containing omega3 fatty-acids on attention-deficit hyperactivity disorder symptoms in children: a double-blind placebo-controlled trial, followed by an open-label extension. Eur Psychiatry. 2012;27(5):335-42.

48. Manor I, Magen A, Keidar D, Rosen S, Tasker H, Cohen $\mathrm{T}$, et al. Safety of phosphatidylserine containing omega 3 fatty acids in ADHD children: a double-blind placebo-controlled trial followed by an open-label extension. Eur Psychiatry. 2013;28(6):386-91.

49. Widenhorn-Müller K, Schwanda S, Scholz E, Spitzer M, Bode H. Effect of supplementation with long-chain $\omega-3$ polyunsaturated fatty acids on behavior and cognition in children with attention deficit/hyperactivity disorder (ADHD): a randomized placebo-controlled intervention trial. Prostaglandins Leukot Essent Fatty Acids. 2014;91(1-2):49-60.

50. Barragán E, Breuer D, Döpfner M. Efficacy and safety of omega-3/6 fatty acids, methylphenidate, and a combined treatment in children with adhd. J Atten Disord. 2014. 